\title{
PROXIMALITY IN MINIMAL TRANSFORMATION GROUPS
}

\author{
LEONARD SHAPIRO
}

Abstract. We present an action of the integers on a compact metric space such that in the resulting minimal topological transformation group the proximal relation is an equivalence relation but is not closed.

The proximal relation in minimal transformation groups with compact Hausdorff phase spaces (here called flows) has been studied in [1], [3], [6]. One of the first problems in this area was whether proximal being an equivalence relation implies it must be closed [1]. We present an example to show that this implication is not true. The construction of our example is similar to the construction of the Morse minimal set described in [8] in that it is a group extension of an almost automorphic flow with the integers as acting group. An adequate reference for the notions involved here is [4]. I wish to thank Professor H. Keynes for many helpful suggestions.

Let $K$ denote the unit circle in the complex plane, $\tau$ a rotation so that $(K, \tau)$ is a minimal flow. Let $k_{1}, k_{2}, \cdots \in K$ so that $O\left(k_{i}\right)$ $=\left\{\left(k_{i}\right) \tau^{n} \mid n \in Z\right\}$ are distinct for distinct $i$, set $E=\bigcup_{i} O\left(k_{i}\right)$ and assume $1 \notin E$. Then there is a minimal flow $(X, \tau)$ described in [7], and a homomorphism $\varphi: X \rightarrow K$ such that $\varphi^{-1}(k)=\left\{k^{+}, k^{-}\right\}$for all $k \in K$ and $k^{+}=k^{-}$iff $k \in K-E$, and such that $k^{+}$is proximal to $k^{-}$for all $k \in K$. We can describe $(X, \tau)$ as the factor space of [4, Example 5.29] after all pairs coming from points in $X-\varphi^{-1}(E)$ have been identified. Two

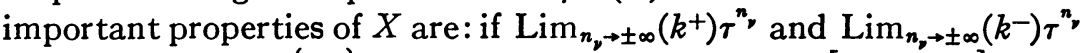
exist for some net $\left\{n_{\nu}\right\}$ in $Z$ then they are equal (cf. [2, p. 613]), and the proximal relation in $X=P(X)=\left\{\left(x_{1}, x_{2}\right) \mid\left\{x_{1}, x_{2}\right\}=\left\{k^{+}, k^{-}\right\}\right.$for some $k \in E$, or $\left.x_{1}=x_{2}\right\}$.

It may be of interest to note that although $(X, \tau)$ is not an expansive flow, it is an inverse limit of expansive flows and a homomorphic image of an expansive flow. We omit the proof of these facts.

Before constructing our example we discuss proximality in an arbitrary group extension of $X$ with trivial bundle.

Let $G$ be a compact abelian group, $f$ a continuous function from $X$

Received by the editors February 5, 1970.

AMS 1969 subject classifications. Primary 5482.

Key words and phrases. Topological dynamics, minimal sets, proximal relation, transformation groups. 
to $G, Y=X \times G, \tau$ the homeomorphism of $Y$ defined by $(x, g) \tau=$ $(x \tau, g(f(x)))$. If $f_{n}(x)=\prod_{i=0}^{n-1} f\left(x \tau^{i}\right)$ for $n \geqq 0$ and $f_{n}(x)=\prod_{i=n}^{-1} f\left(x \tau^{i}\right)$ for $n<0$ then $(x, g) \tau^{n}=\left(x \tau^{n}, g\left(f_{n}(x)\right)\right)$. Define:

$$
\begin{aligned}
\rho_{i}(k) & =f\left(k^{+} \tau^{i}\right)\left[f\left(k^{-} \tau^{i}\right)\right]^{-1}, \\
\sigma_{n}(k) & =\prod_{i=0}^{n-1} \rho_{i}(k)=f_{n}\left(k^{+}\right)\left[f_{n}\left(k^{-}\right)\right]^{-1} \text { for } n \geqq 0, \\
\sigma_{n}(k) & =\prod_{i=n}^{-1}\left[\rho_{i}(k)\right]^{-1}=f_{n}\left(k^{-}\right)\left[f_{n}\left(k^{+}\right)\right]^{-1} \text { for } n<0 .
\end{aligned}
$$

Then $(Y, \tau)$ is a (not necessarily minimal) flow, and $\pi: Y \rightarrow X$ defined by $\pi(x, g)=x$ is a homomorphism of flows.

Lemma 1. Assume $\left(x_{1}, g_{1}\right)$ is proximal to $\left(x_{2}, g_{2}\right)$. If $x_{1} \neq x_{2}$, then $\left\{x_{1}, x_{2}\right\}=\left\{k^{+}, k^{-}\right\}$for some $k \in K$, and if $x_{1}=x_{2}$ then $g_{1}=g_{2}$.

PROOF. If $x_{1} \neq x_{2}$ and $\left(x_{1}, g_{1}\right)$ is proximal to $\left(x_{2}, g_{2}\right)$ then $\pi\left(x_{1}, g_{1}\right)=x_{1}$ is proximal to $\pi\left(x_{2}, g_{2}\right)=x_{2}$ so $\left\{x_{1}, x_{2}\right\}=\left\{k^{+}, k^{-}\right\}$for some $k \in K$. If $x_{1}=x_{2}$ then let $\left\{n_{\nu}\right\}$ be such that $\operatorname{Lim}_{\nu}\left(x_{1}, g_{1}\right) \tau^{{ }^{{ }_{\nu}}}=\operatorname{Lim}_{\nu}\left(x_{1}, g_{2}\right) \tau^{{ }^{n}}$. Considering second coordinates we have $\operatorname{Lim}_{\nu} g_{1} f_{n_{\nu}}\left(x_{1}\right)=\operatorname{Lim}_{\nu} g_{2} f_{n_{\nu}}\left(x_{1}\right)$, and letting $g_{0}=\operatorname{Lim}_{v} f_{n_{v}}\left(x_{1}\right)$ we have $g_{1} g_{0}=g_{2} g_{0}$ so $g_{1}=g_{2}$.

LEMMA 2. $P(Y)$ is an equivalence relation iff $\operatorname{Lim}_{|n| \rightarrow \infty} \sigma_{n}(k)=\xi(k)$ exists for each $k \in K$.

Proof. Suppose $\xi(k)$ exists. Let $k^{+} \neq k^{-}$and suppose $\left(k^{+}, g_{1}\right)$ is proximal to $\left(k^{-}, g_{2}\right)$. We will show that $g_{2}=g_{1} \xi(k)$ and this with Lemma 1 then will imply that $P(Y)$ is an equivalence relation. Let $\left\{n_{\nu}\right\}$ be such that $\operatorname{Lim}_{\nu}\left(k^{+}, g_{1}\right) \tau^{n_{\nu}}=\operatorname{Lim}_{\nu}\left(k^{-}, g_{2}\right) \tau^{n_{\nu}}$, then $\operatorname{Lim}_{\nu} g_{1} f_{n_{\nu}}\left(k^{+}\right)$ $=\operatorname{Lim}_{\nu} g_{2} f_{n_{\nu}}\left(k^{-}\right)$so

$$
g_{2}=g_{1} \operatorname{Lim}_{n_{\nu}}\left(k^{+}\right)\left[f_{n_{\nu}}\left(k^{-}\right)\right]^{-1}=g_{0} \xi(k) .
$$

Conversely, suppose there are nets $\left\{n_{\nu}\right\}$ and $\left\{m_{\nu}\right\}$ and $k \in K$ such that

$$
g_{1}=\operatorname{Lim}_{\nu} \sigma_{n_{\nu}}(k) \neq \operatorname{Lim}_{\nu} \sigma_{m_{\nu}}(k)=g_{2}
$$

Since $K$ is compact we may assume that $\left\{k^{+} \tau^{n} v\right\}$ and $\left\{k^{-} \tau^{n} v\right\}$ converge, thus they converge to the same point, and similarly for the net $\left\{m_{\nu}\right\}$. Then $\left(k^{+}, e\right)$ ( $e$ is the identity of $\left.G\right)$ is proximal to $\left(k^{-}, g_{1}\right)$ (along the net $\left.\left\{n_{\nu}\right\}\right)$ and to $\left(k^{-}, g_{2}\right)$ (along $\left\{m_{\nu}\right\}$ ). By Lemma 1, $\left(k^{-}, g_{1}\right)$ is not proximal to $\left(k^{-}, g_{2}\right)$ so $P(Y)$ is not an equivalence relation. 
CoROLlaRY 1. $\xi(k)$ exists iff for each $g \in G$ there is a unique $g_{1} \in G$ such that $\left(k^{+}, g\right)$ is proximal to $\left(k^{-}, g g_{1}\right)$. If $\xi(k)$ exists then $g_{1}=\xi(k)$. If $\xi(k)$ exists then $\xi\left(k \tau^{m}\right)$ exists for all $m$.

PRoof. The first two statements follow from the proof of Lemma 2. The third follows from the first because $P(Y)$ is invariant under $\tau$.

Lemma 3. Assume $\xi(k)$ exists for all $k \in K$. Then $P(Y)$ is closed iff: $\operatorname{Lim}_{\nu} \xi\left(k_{\nu}\right)=g$ for $\left\{k_{\nu}\right\}$ a net of distinct elements implies $g=e$.

Proof. Suppose the condition holds. By Corollary 1, in order to show $P(Y)$ is closed we need only prove the following: suppose $\left(k_{\nu}^{+}, g_{v}\right)$ is proximal to $\left(k_{\nu}^{-}, g_{\nu} \xi\left(k_{v}\right)\right)$ and $\left(x_{1}, g_{1}\right)=\operatorname{Lim}_{\nu}\left(k_{\nu}^{+}, g_{v}\right),\left(x_{2}, g_{2}\right)$ $=\operatorname{Lim}_{\nu}\left(k_{\nu}^{-}, g_{\nu} \xi\left(k_{\nu}\right)\right)$, then $\left(x_{1}, g_{1}\right)$ and $\left(x_{2}, g_{2}\right)$ are proximal. Since $\operatorname{Lim}_{\nu} g_{\nu}=g_{1}$ and $\operatorname{Lim}_{\nu} g_{\nu} \xi\left(k_{\nu}\right)=g_{2}$ we must have $g_{2}=g_{1} \operatorname{Lim}_{\nu} \xi\left(k_{\nu}\right)$. If the $\left\{k_{\nu}\right\}$ are distinct then $x_{1}=x_{2}$ and by our assumption $\operatorname{Lim} \xi\left(k_{v}\right)=e$ and $g_{2}=g_{1}$. If the $k_{v}$ are all equal to a certain $k$, then $\left(x_{1}, g_{1}\right)=\left(k^{+}, g_{1}\right)$ and $\left(x_{2}, g_{2}\right)=\left(k^{-}, g \xi(k)\right)$ and we invoke Corollary 1 to see that these points are proximal.

Conversely, let $\left\{k_{v}\right\}$ be distinct elements of $K$ with $\operatorname{Lim}_{\nu} \xi\left(k_{v}\right)$ $=g_{1} \neq e$. We may assume $\operatorname{Lim}_{\nu} k_{\nu}^{+}=\operatorname{Lim}_{\nu} k_{\nu}^{-}=x_{1}$. If $P(Y)$ is closed then $\left(x_{1}, e\right)=\operatorname{Lim}_{v}\left(k_{\nu}^{+}, e\right)$ is proximal to $\operatorname{Lim}_{v}\left(k_{\nu}^{-}, \xi\left(k_{v}\right)\right)=\left(x_{1}, g_{1}\right)$, contradicting Lemma 1.

Now to define $f$ : define an order on $K-\{1\}$ by: if $k, m \in K$, then $k \geqq m$ if 1 is not a member of $(k, m)$, the arc traversed clockwise from $k$ to $m$, and define $\left[k^{+}, m^{-}\right]=\phi^{-1}((k, m)) \cup\left\{k^{+}, m^{-}\right\}$. Then the intervals $\left[k^{+}, m^{-}\right]$are clopen sets. Pick $G$ to be the complex numbers of modulus 1. If $n \geqq 1$ pick $p(n, j) \in Z$ for $j= \pm 1, \pm 2, \cdots, \pm n$ and define $k_{n, j}=\left(k_{n}\right) \tau^{p(n, j)}$ so that

(a) $p(n, j) \gtrless 0$ if $j \gtrless 0$,

(b) $\operatorname{Lim}_{n \rightarrow \infty} k_{n, i}=1$ for all $i$,

(c) $k_{1,1}<k_{2,1}<\cdots<k_{n-1, n-1}<k_{n, 1}<k_{n, 2}<\cdots<k_{n, n} \cdots$ and $k_{n,-n}<\cdots<k_{n,-2}<k_{n,-1}<k_{n-1,-(n-1)}<\cdots$ $<k_{1,-1}<k_{1,1}$.

These choices can be made because $O\left(k_{n}\right)$ is dense for each $n$. Define:

$$
\begin{aligned}
A_{0} & =\left\{\left[k_{1,-1}^{+}, \overline{k_{1,1}}\right]\right\}, \text { and for } n \geqq 1, \\
A_{n} & =\left\{\left[k_{n, 1}^{+}, k_{n, 2}^{-}\right],\left[k_{n, 2}^{+}, k_{n, 3}^{-}\right], \cdots,\left[k_{n, n}^{+}, k_{n+1,1}^{-}\right]\right\} \text {and } \\
A_{-n} & =\left\{\left[k_{n+1,-1}^{+}, k_{n,-n}^{-}\right],\left[k_{n,-n}^{+}, k_{n,-n+1}^{-}\right], \cdots,\left[k_{n,-2}^{+}, k_{n,-1}^{-}\right]\right\} .
\end{aligned}
$$


Note that by (1)(b), $X-\cup\left\{A_{m} \mid m \in Z\right\}=\{1\}$. Set $\epsilon_{m}=\exp (\sqrt{ }(-1) / m)$ $\in G$ and define $f: X \rightarrow G$ by

(a) $f(x)=1$ for $x \in\left[k_{1,-1}^{+}, k_{1,1}^{-}\right]$.

(b) If $[a, b] \in A_{m}$ and $k^{+}, k^{-} \in[a, b]$ then require $f\left(k^{+}\right)=f\left(k^{-}\right)$. After this identification $[a, b]$ is homeomorphic to a closed arc in $K$, and we map it to $\left[\epsilon_{m}, 1\right]$ (clockwise arc from $\epsilon_{m}$ to 1) if $m \geqq 1$ and to $\left[1, \epsilon_{m}\right]$ if $m \leqq-1$, with $f(a)=\epsilon_{m}, f(b)=1$ if $m \geqq 1$, and $f(a)=1, f(b)=\epsilon_{m}$ if $m \leqq-1$.

(c) $f(1)=1$.

Since the intervals in the $A_{m}$ are all distinct and clopen $f$ is continuous on $U\left\{A_{m} \mid m \in Z\right\}$, and $f$ is continuous at 1 by inspection, so $f$ is continuous.

We make a number of observations, which lead to the fact that $P(Y)$ is an equivalence relation but is not closed: (a) $f\left(k^{+}\right)=f\left(k^{-}\right)$if $k \neq k_{n, j}$ for some $n, j$; (b) By (3) (b), if $i=p(n, j)>0$ then $\rho_{i}\left(k_{n}\right)=f\left(k_{n, j}^{+}\right)$ $\left[f\left(k_{n, j}^{-}\right)\right]^{-1}=\epsilon_{n} ;$ (c) Similarly if $i=p(n, j)<0$ then $\rho_{i}\left(k_{n}\right)=\epsilon_{-n}$; (d) By (a), $\rho_{i}\left(k_{n}\right)=e$ for $i \neq p(n, j)$ for all $n, j$; (e) By (b) and (d) if $m>p(n, j)$ for all $j$ then $\sigma_{m}\left(k_{n}\right)=\left(\epsilon_{n}\right)^{n}=\epsilon_{1}$; (f) By (c) and (d) if $m<p(n, j)$ for all $j$ then $\sigma_{m}\left(k_{n}\right)=\left(\left(\epsilon_{-n}\right)^{-1}\right)^{n}=\epsilon_{1}$; (g) By (e) and (f), $\xi\left(k_{n}\right)=\epsilon_{1}$ for all $n$; (h) By (g) and Corollary $1, \xi(k)$ exists for $k \in E$; (i) If $k \notin E$ then $k \neq k_{n, j}$ for all $n, j$ so, by (a), $\rho_{i}(k)=e$ for all $i$ and $\xi(k)=e$.

By (h) and (i), $\xi(k)$ exists for all $k$ so $P(Y)$ is an equivalence relation. $\operatorname{Lim}_{n \rightarrow \infty} \xi\left(k_{n}\right)=\operatorname{Lim}_{n \rightarrow \infty} \epsilon_{1} \neq e$ so $P(Y)$ is not closed.

In our example $\pi: Y \rightarrow X$ is a group extension [5, Definition II.4] by the group $G$ if we take the action of $g_{0} \in G$ on points $(x, g) \in Y$ to be $(x, g) \rightarrow\left(x, g g_{0}\right)$. As in any flow, there must be a minimal subset $M$ of $Y$. (In fact $Y$ is a union of minimal subsets by $[5$, p. 31].) The next lemma shows that such an $M$ provides our desired example.

Lemma 4. Let $(X, \tau)$ be minimal, and let $(Y, \tau)$ be a group extension of $(X, \tau)$ by the compact group $G$. If $P(Y)$ is an equivalence relation which is not closed then the same is true of $P(M)$, for any minimal subset $M$ of $Y$.

Proof. Let the extension be denoted by $\pi: Y \rightarrow X$ so $\pi(y)=\pi\left(y^{\prime}\right)$ iff $y^{\prime}=y g$ for some $g \in G$. Let $M$ denote a minimal subset of $Y$.

If $y, y^{\prime} \in Y$ are proximal and $y \in M$ then $y^{\prime}$ is in the orbit closure of $y$, which is $M$. Thus $P(M)$ is an equivalence relation if $P(Y)$ is.

Now $\pi(M)=X$ since $X$ is minimal, so if $y \in Y$ there must be $g \in G$ with $y g \in M$. Assuming $P(Y)$ is not closed there are $\left(y_{p}, y_{v}^{\prime}\right) \in P(Y)$ with $\operatorname{Lim}_{\nu}\left(y_{v}, y_{\nu}^{\prime}\right) \notin P(Y)$. Choose $g_{\nu} \in G$ so that $y_{\nu} g_{\nu} \in M$ then $y_{\nu}^{\prime} g_{\nu}$, being 
proximal to $y_{\nu} g_{\nu}$ (since $\tau$ commutes with the action of $G$ ), will be in $M$ by the preceding paragraph, so $\operatorname{Lim}_{\nu}\left(y_{\nu} g_{\nu}, y_{\nu}^{\prime} g_{\nu}\right)=\left(y g, y g^{\prime}\right) \oplus P(M)$, so $P(M)$ is not closed.

\section{BIBLIOGRAPHY}

1. J. Auslander, On the proximal relation in topological dynamics, Proc. Amer. Math. Soc. 11 (1960), 890-895. MR 29 \#1632.

2. - Endomorphisms of minimal sets, Duke Math. J. 30 (1963), 605-614. MR 27 \#5245.

3. J. Clay, Proximity relations in transformation groups, Trans. Amer. Math. Soc. 108 (1963), 88-96. MR 27 \#4218.

4. R. Ellis, Lectures on topological dynamics, Benjamin, New York, 1969.

5. H. Furstenberg, Disjointness in ergodic theory, minimal sets, and a problem in Diophantine approximation, Math. Systems Theory 1 (1967), 1-49. MR 35 \#4369.

6. H. Keynes, On the proximal relation being closed. Proc. Amer. Math. Soc. 18 (1967), 518-522. MR 35 \#3645.

7. L. Shapiro, The structure of $H$-cascades, Cleveland Conference in Global Differentiable Dynamics (to appear).

8. W. Veech, Strict ergodicity in zero dimensional dynamical systems and the Kronecker-Weyl theorem mod 2, Trans. Amer. Math. Soc. 140 (1969), 1-33. MR 39 \#1410.

University of Minnesota, Minneapolis, Minnesota 55455 